\title{
Optimization of ALD ZnO for Antibacterial Activity
}

\author{
Beom Joon Kim¹, Kang-Hee Park ${ }^{1}$, Gwon Deok Han², Joon Hyung Shim ${ }^{2}$, Hee-Deung Park ${ }^{1}$ \\ ${ }^{1}$ School of Civil, Environmental and Architectural Engineering, Korea University \\ 145, Anam-Ro, Seongbuk-Gu, Seoul 02841, South Korea \\ goodmaner778@naver.com; newpkh@korea.ac.kr; heedeung@korea.ac.kr \\ ${ }^{2}$ School of Mechanical Engineering, Korea University \\ 145, Anam-Ro, Seongbuk-gu, Seoul, South Korea \\ pranchisco@korea.ac.kr; shimm@korea.ac.kr
}

\section{Extended Abstract}

There are many microorganisms which are potentially harmful for human in the polluted water. As these microorganisms can cause diseases to human, it is needed to treat them using a proper method. Metal oxides such as $\mathrm{ZnO}$, $\mathrm{TiO}_{2}, \mathrm{AgO}$ have been reported to show antibacterial effects when they are exposed to UV light, although antibacterial effects are different depending on surface properties of the metal oxides. [2] An important factor for determining the antibacterial efficiency is reactive surface area. To enhance the reactive surface area, in our previous study, glass slides was coated with $\mathrm{ZnO}$ using atomic layer deposition (ALD). [1] A benefit using the $\mathrm{ZnO}$ thin films is no need for recycling of metal oxides after water treatment and require a lower light energy compared with conventional lights for killing microorganisms. We also demonstrated that glass slides coated with $\mathrm{ZnO}$ films by ALD were effective to produce reactive oxygen species and to kill a model microorganism. Nevertheless, the optimum condition of $\mathrm{ZnO}$ thin film formation has not been studied. Therefore, the main objective of this study was to investigate the optimum condition for maximizing antibacterial effects.

There are two main parameters affecting the efficiency of antibacterial property of the ALD ZnO films: ALD cycles and synthesis temperature. In this study, $\mathrm{ZnO}$ thin films were formed on glass slides. ZnO films were synthesized under four different synthesis temperature conditions $\left(100^{\circ} \mathrm{C}, 150^{\circ} \mathrm{C}, 200^{\circ} \mathrm{C}\right.$, and $\left.250^{\circ} \mathrm{C}\right)$ and two different ALD cycles $(100$ cycles and 400 cycles), respectively. Conditions of the synthesis temperature and the ALD cycles are currently optimized. [3] The antibacterial activity of ALD $\mathrm{ZnO}$ decreases when the synthesizing temperature was about $250^{\circ} \mathrm{C}$. Moreover, the antibacterial activity of different cycle (100 cylces and 400 cycles) has little difference. This study will provide the mechanisms of ROS productions in association with antibacterial properties for various $\mathrm{ZnO}$ thin films formed by ALD.

\section{References}

[1] K.-H. Park, "Antibacterial activity of the thin $\mathrm{ZnO}$ film formed by atomic layer deposition under UV-A light," Chemical engineering journal., vol. 328, pp. 988-996, 2017.

[2] Y. Li, "Mechanism of Photogenerated Reactive Oxygen Species and Correlation with the Antibacterial Properties of Engineered Metal-Oxide Nanoparticles," ACS NANO., vol. 6, no. 6, pp. 5164-5173, 2012.

[3] S.-J. Park, "Comparing the toxic mechanism of synthesized zinc oxide nanomaterials by physicochemical characterization and reactive oxygen species properties,” Toxicology Letters., vol. 207, pp. 197-203, 2011. 